\title{
Accessing social service support: Barriers experienced by Chinese migrants living in Auckland, Aotearoa New Zealand
}

\author{
King Lok Kan', Helene Connor ${ }^{2}$ and Liz Beddoe ${ }^{3}$
}

\begin{abstract}
INTRODUCTION: According to Census 2013, approximately 25\% of Auckland's population is Asian, with the majority of these being Chinese. Given that Aotearoa New Zealand's population is ageing, it can be expected that there will a significant demand for aged services for Chinese older adults in our local community in the future. Exploring the language barrier encountered by retired and aged (65+) Chinese migrants living in Auckland is needed.
\end{abstract}

METHOD: Two focus groups were interviewed with retired Chinese migrants (group 1) and local social service practitioners (group 2). The focus group topics included the living experience of Chinese migrants in Auckland in Aotearoa New Zealand, while local social service practitioners reported their daily work experience of serving Chinese older adults. A short, warm-up, oral questionnaire stimulated discussion in each group. Data analysis was used to identify themes and draw tentative conclusions about the needs of older migrants and suggest how local agedservices providers could better serve retired Chinese migrants.

FINDINGS: Language barriers and transportation problems were commonly agreed to be obstacles that hindered retired Chinese migrants accessing social support and health services. More input was needed to improve their general English language proficiency to facilitate their daily lives and participation in social life in Aotearoa New Zealand.

IMPLICATIONS: Findings from the research have the potential to be an important resource for local social service practitioners, giving them a better understanding of older Chinese people.

KEYWORDS: Retired Chinese migrants; quality of life; social support; barriers to accessing social support; immigration; aged service

Since the 2000s, Aotearoa New Zealand has had a significant increase in population from Chinese migration. Auckland, as the biggest city in Aotearoa New Zealand, is the main place migrants choose to settle. As a result, Auckland has become a superdiverse city and is now home to speakers of 160 languages, with this population diversity likely to increase over the next decade (Statistics New Zealand), 2013). Statistics
New Zealand (2013) reports there are seven common languages spoken by multilingual people in Auckland region: English, Samoan, Hindi, Northern Chinese, Mãori, Yue and Tongan. Some of these languages have different dialects that may hinder communication-for example, Yue is spoken differently between Hong Kong and Guangzhou, and Mandarin differently between Taiwan and Mainland China.
${ }^{1}$ Ara Institute of Canterbury

${ }^{2}$ Te Puna Wananga, University of Auckland

${ }^{3}$ Counselling, Human Services and Social work, University of Auckland
AOTEAROA NEW ZEALAND SOCIAL WORK 32(4), 131-144.

CORRESPONDENCE TO: King Lok Kan lok.kan@ara.ac.nz 
The increased number of Chinese migrants has resulted in "Asian" becoming the second largest ethnic group in Aotearoa New Zealand, after European (Stats NZ, 2017). Apart from the "European and Other" groups, the Asian ethnic group is forecast to surge the most between 2013 and 2038, with its population projected to grow from 540,000 in 2013 to about 1.4 million by 2038 . By 2023, it is expected that there will be more Asian Aucklanders than Māori Aucklanders, and it is anticipated that Asians will make up $22 \%$ of the total number of people in Auckland by 2038, followed by Europeans and others. In other words, it is foreseeable that a larger number of immigrants from China will be concentrated in Auckland (Li, 2014).

At the same time, it should be noted that ageing populations are a critical issue throughout the world. In Aotearoa New Zealand, $14.3 \%$ of the population was aged over 65 years at the time of the 2013 Census, and this proportion is projected to rise to about 26.7\% in 2063 (Statistics NZ, 2015). With the increasing number of Chinese migrants living in Auckland, social services for Chinese migrants aged $65+$ will be one of the future challenges facing social workers and other social service practitioners in this country.

Communicating in English is one of the most common difficulties facing migrants from non-English-speaking countries; it is also a common indicator of how well a migrant is adapting to their new cultural environment (Wang, Schwartz, \& Zamboanga, 2010).This study investigated the language barriers retired Chinese migrants aged 65+ face accessing social support in Aotearoa New Zealand; exploring the perceptions of local social service practitioners concerning delivering services to retired Chinese migrants. Its findings highlight concerns about the current effectiveness of agedcare services in New Zealand and so help to increase the general well-being of aged people living in this country.

\section{Background}

Auckland is New Zealand's largest city and has the largest proportion of migrants in the world. In 2009, about $40 \%$ of Auckland's population were immigrants (Spoonley \& Butcher, 2009). From 2000 to 2008, over 63,000 Chinese residents were granted permanent residence. Of these, some had moved to Aotearoa New Zealand via business investment migration categories. According to statistics from Immigration New Zealand, Chinese applicants made up nearly $75 \%$ (7286 out of 9672) of the business investment migrants approved. Many Chinese students who completed their three- to four-year study in New Zealand were granted permanent residency. At the same time, there was a gradual increase in the number of Chinese "Family Parent" migrants to New Zealand. According to Department of Labour figures, China had more permanent residence approvals under the "Family Parent" scheme between 2004 and 2017 than any other country (see Table 1). The ratio of approvals to Chinese migrants rose significantly from $21 \%$ in $2004 / 05$ to $55 \%$ in $2011 / 12$, and then to $60 \%$ in $2016 / 17$. Although this pathway ceased in

Table 1. New Zealand Permanent Residence Approved Through Parent Category: 2004/05 to 2016/17

\begin{tabular}{|l|c|c|c|c|c|c|c|}
\hline Year & $2004 / 05$ & $2005 / 06$ & $\ldots \ldots .$. & $2011 / 12$ & $2012 / 13$ & $\ldots . .$. \\
\hline Total & 2776 & 4400 & & 4601 & 3840 & 1820 \\
\hline China & 583 & 1232 & & 2171 & 1684 & 1092 \\
\hline$\%$ & $21 \%$ & $28 \%$ & & $47 \%$ & $43 \%$ & $60 \%$ \\
\hline Ranking & 1 & 1 & & 1 & 1 & 1 \\
\hline
\end{tabular}

Source: Ministry of Business, Innovation and Employment, Migration Trends and Outlook 2004/05-2016/17. 
2016, it has brought a great number of older Chinese people to New Zealand.

The coverage and effectiveness of related social support and aged services for this large number of retired Chinese migrants will become a critical issue, not just in Auckland, but the whole country, because it will directly affect the retired migrants' wellbeing. To enhance the level of social support for retired Chinese migrants, it is necessary to explore the difficulties they face accessing social support and also the barriers to local aged-services practitioners serving retired Chinese migrants.

\section{Literature review}

Acculturation strategies theory suggests that immigrants adapting to a new environment need to deal with the potential struggle between the person's own culture and that of the mainstream (Berry, Phinney, Sam, \& Vedder, 2006). Some of the conflicts can be easily settled, like changing one's eating habits, clothing and certain behaviours, while some cannot, and will lead to different levels of acculturative stress (Berry et al., 2006).

Research conducted in Australia reported that that elderly Chinese migrants in Brisbane is relevant to the concerns that motivated this current Auckland study of older Chinese (Ip, Lui, \& Chui, 2007). Ip et al. conducted a community survey and five focus groups, three with different groups of older people in the Chinese community of Brisbane, one with adult children of older Chinese, while the fifth group included service providers, community leaders and health-care practitioners. The older ChineseAustralians and adult-children groups explored perceived and relative needs, while the service providers were encouraged asked "to concentrate on normative and expressed needs" (p. 724). Ip et al. found that half of their survey informants lived with their adult children, while all the older Chinese focus group participants were living with their adult children and their social networks were very restricted, especially the women's. More commonly, participants had little contact with their friends, with nearly one third reporting they seldom or never met their friends. Some older adults also reported that they felt uncomfortable using the phone for cost reasons or because they were worried about being seen as preventing incoming calls to others in the household (Ip et al., 2007).

Language and mobility problems are also behind retired Chinese migrants' social isolation. The Brisbane survey found that participants generally had a poor command of English even though $42 \%$ had completed secondary education or higher (Ip et al., 2007). A sizeable proportion of the female participants were not able to write and speak English and none considered herself capable of writing or speaking English effectively. Although many had enrolled in English language classes, they had usually quickly dropped out because they found it very hard to learn a new language. They explained their belief that their age made it difficult for them to learn new things (Ip et al., 2007). The language problem negatively impacted on their confidence to go out alone-for example, to visit friends or take part in social activities-which, in turn, lowered their self-esteem and worsened their social isolation. This had seriously restricted their communication with non-Chinese speakers and stopped the retired Chinese migrants from participating in special events and programmes for aged people organised by Brisbane City Council (Ip et al., 2007).

The experiences reported by Ip et al. can also be found in New Zealand. For example, professionals anecdotally note that older Chinese, when they are ill, prefer to consult Chinese-speaking doctors and can be severely disadvantaged when accessing health and other social services. They find it difficult to access information about free services and amenities for older people because most of these are advertised in English (K. Quan, personal communication, February 11, 2019). A medical practitioner 
in the Brisbane survey mentioned that it is hard to find specialists who can speak both Chinese and English (Ip et al., 2007). And, although a qualified interpreter can interpret effectively and accurately to Chinese patients about their medical conditions, many retired Chinese insist on relying on their adult children to provide interpretation and their children have difficulties understanding medical jargon. This confusion can result in the need for additional visits, which cost both time and money (Ip et al., 2007).

The results from the Brisbane survey revealed that the language barrier experienced by retired Chinese migrants living in a foreign land leads to many different problems in their daily living, although the migrants do not all experience the same problems (Ip et al., 2007). For example, retired Chinese migrants find it difficult to get involved in local groups and are disengaged with social support services because such services are not commonly found in China. No local research in Auckland could be found that explored how different languages available in social services influences aged-care delivery to elderly Chinese migrants. Therefore, this study aimed to explore these concerns within the Auckland setting, and in doing so, the study's findings will provide a valuable reference for both policymakers and service providers in Aotearoa New Zealand.

\section{From social support for older people to the quality of life}

Cummins (2005) suggested that a range of elements, from general to specific, make up the concept of quality of life. They are common to all people regardless of their culture and socio-economic status; for example, individual health. Specific components, however, can vary between different people. Farquhar (1995) found that many older people agreed that connections with others or the community are significant to their quality of life, while Higgs et al. (2005) found that older people have a tendency to rate how healthy they are as more important than how many assets they own. Other scholars have also discovered that support from family and the neighbourhood are more important to ethnic minorities than to majority populations (Bajekal, Blane, Grewal, Karlsen, \& Nazroo, 2004; Chappell, 2007; Remennick, 2003). It is argued that the quality of life of older Chinese migrants should be explored within the migration context because they face significant changes to their living environment and are exposed to different cultures when they relocate, and these changes potentially have a critical impact on elderly migrants' perceptions of their quality of life. As social support is an important component of helping retired Chinese migrants manage the difficulties of living in an unfamiliar environment, it is necessary to investigate the relationship between between language difficulties that complicate accessing social support and achieving a quality of life.

\section{Method}

The aim of this study was to explore the language barriers that may be encountered by retired and aged $65+$ Chinese migrants, living in Auckland who wish to participate in their local community. In addition, the perceptions of aged-services practitioners serving retired Chinese migrants about their needs were sought. Qualitative methods were used to enable these research participants to voice their subjective perceptions of social support. This methodology allows the researcher to identify the most influential components in social support and evaluate the elderly Chinese migrants' overall "satisfactory" level of social support. Interviewing the practitioners' group also provides an opportunity to identify the most critical obstacles that reduce the overall effectiveness of their aged-services delivery. The use of interviews enables broader investigation of the experiences the participants have run into, recognition of their understandings of social support in their migrant context in a new country, and clarification of the 
differences between service receivers (the elderly Chinese migrants) and service providers (the aged-services practitioners).

This article is based on a research study completed and submitted for a Master of Social Work degree at the University of Auckland. Ethical approval for the study was sought from the University of Auckland Human Participants Ethics Committee, and approval given for the study to proceed.

Five participants in the Chinese migrants' group were recruited with the assistance of Age Concern Auckland, while the five participants in the practitioners' group were staff members of Age Concern Auckland. The criteria employed to recruit the elderly Chinese migrants were: they had migrated from Mainland China at least one year ago; were aged 65 or above and retired. Those for the social workers or practitioners were: born in Aotearoa New Zealand; practising in aged service; and involved in serving or contacting Chinese clients.

The two groups were interviewed separately in suitable, comfortable community locations. The Participant Information Sheet was provided in Mandarin and Cantonese. The broad questions were indicated on the information sheets. Table 2 sets out the topics explored in the focus groups.

The focus groups took between 40 and 60 minutes. All focus groups were digitally recorded and transcribed. The data were then coded and simple thematic analysis were applied. Post-analysis translation was also done in order to help to avoid any distortion of meanings missed when data were translated from Chinese into English.
Four women and one man, all aged between 68 and 75, were invited to participate in the focus group of Chinese migrants. All of them had migrated to New Zealand from China (one from Hong Kong) more than a year prior to the focus groups.

Three women and two men from social service Age Concern Auckland participated in the focus group of practitioners. Four were European and one was a Pacific Islander. They had all been practising in aged service for more than a year. English is the main language of their daily work and none of them can speak a Chinese language.

\section{Findings}

Several main themes were developed from the transcribed interviews. English language proficiency mobility and transportation, financial concerns and related to barriers to social participation were identified in both the experiences of the retired Chinese migrants and the perceptions of needs identified by the local practitioners.

\section{The retired Chinese migrants}

Four of the five retired Chinese migrants reported that they had experienced serious communication problems in their everyday lives in Auckland. Adapting to an Englishspeaking country has been difficult for them, although they had arrived in Auckland between five and 20 years ago.

The study participants said they had unsuccessfully searched for English language classes, which implies that the support available is not effectively linked to the targeted people. Although some information

Table 2. Focus Group Topics

Group of Participants

Chinese Migrants' Group, age 65+

Aged-services Practitioners' Group
Area explored

Barriers of accessing social support including language, mobility, financial problems, resources and recreation needs.

The experience of providing services to Chinese migrants and the perceptions of the needs of aged services. 
can be found at Citizens Advice Bureaus, retired Chinese migrants felt uncomfortable seeking help or information from outside of their Chinese community. Even if plenty of information can be found online, none of this information was available in a Chinese language. Thus, the retired Chinese migrants in this study reported a lack of support for them to learn the English language.

Chinese migrant participants reported that the language barrier also contributed significant or moderate difficulties in travelling around the city. Due to the challenge of driving in Auckland with unfamiliar road rules and navigation concerns, all the participants reported regular use of public transport. However, their responses indicated that they were unhappy with Auckland's public transport system as it was not meeting their needs and thus posed a very significant barrier to their well-being. Older migrant participants faced difficulty if they became lost in Auckland because they could not communicate with public transport staff in English.

As part of their group interview session, the elderly Chinese migrants discussed how the language barrier affects different areas in their daily lives; for example, when accessing health services:

Shopping in a grocery normally does not involve any language problems because you just need to pay money and no need to speak anymore. But seeing a doctor or living in a hospital will be a big problem. I cannot understand their questions and then I cannot answer them correctly, such as asking me if I have any food allergy. (Mary, 70)

While new technology offers many smartphone aids to improve daily life, it was apparent that not every retired Chinese migrant was able to use smart devices to assist their daily life. Some were still struggling with the language barrier even after residing in Auckland for up to 20 years. For example:
Communication with others in English language is nearly impossible, but just for everyday life is still fine and I will try to express the meaning by body language if necessary. (Susan, 72)

Participant 4 said there is very little difference in their English language ability now compared with when they first arrived in Auckland:

We [with male participant 5] are just learning it step by step slowly and word by word, and easy to forget what I have learnt easily. You know, for an old age person like me, learning a new language is not an easy thing. I think I have spent at least five years to make myself feel a very little difference ... hearing or understanding a little from others. But I still find hard to tell the others what I am thinking about. (Tracy, 68)

Nearly all the participants expressed that it would be impossible for them to live in any cities outside Auckland where there are not sizeable Chinese populations. For example:

It is nearly impossible for me to live in another city without a sized Chinese community. In Auckland, we can find Mandarin-speaking staff in bank, Chinese grocery. Without these staff, it would be much more difficult for us to live. (Tony, 74)

During the group discussion, the participants talked about what made their everyday life in Auckland difficult. Interestingly, they discussed their expectations for improvement in family and home support services:

Family support services should be improved because many old people are living alone. This is particularly high risk for people who do not speak English language. Nobody can acknowledge the accidents and ... older people cannot call 111 by themselves because of the language barrier. (Tracy, 68) 
The participants' comments indicate that the mobility of retired Chinese migrants is limited by Auckland's disappointing public transport system. One of the study participants from East Auckland said she would not go to other parts of Auckland unless she needs to see her family doctor in the west. There was a feeling that English language training should be provided by the New Zealand Government to tackle the communication difficulties migrants from non-English-speaking backgrounds experience:

I think the New Zealand Government can offer more support to the non-Englishspeaking migrants like us to improve their English language. In China, many Western people working in Beijing and Shanghai can speak Mandarin very well and they live in China very well although Mandarin is hard to learn. Thus, I hope the local government can employ more English teachers, who can speak Mandarin also, to make us learn the English language easier. We only expect to pick up some basic level of English language for daily living. You can predict ... how much ... money the Government can save from offering the translation service to us if we can manage the communication problem by ourselves. (Tony, 74)

\section{The practitioners}

Although all five aged-services practitioners indicated that they find it difficult, or very difficult, to serve retired Chinese migrants, their responses indicated that only one of the participants works with retired Chinese migrants regularly. This practitioner felt it was very difficult to serve retired Chinese migrants because they generally have no idea about the service systems in Aotearoa New Zealand. One practitioner explained that she needed to take much more time to explain what is happening in each stage of the service.

Generally, the aged-services practitioners perceive the social connection of the retired
Chinese migrant to be moderate. Also, they thought that retired Chinese migrants would find it difficult to integrate into the local community even if they are actively involved in their Chinese community, because retired Chinese migrants who cannot speak English are much more likely to experience difficulty accessing social support in Auckland.

Some of the practitioners recognised that support for those working with retired Chinese migrants is insufficient because there are not enough Mandarin- or Cantonese-speaking social workers or support workers in their organisations. As a result, Chinese staff are burning out.

They're [social workers] burning up because of the demand for the service, whatever it may be-translationwhatever it may be ... the demand is just ever-increasing and unless we increase the amount of services, those people are just gonna, I think, burn out all the way and something else. It's just too much. (Christine)

In addition to Mandarin- and Cantonesespeaking staff having unfair expectations placed upon them, the lack of long-term funding was mentioned as another reason practitioners feel unsupported:

There's not enough support within the organisation to support them [staff in the Asian service team], and it could be a really, really difficult situation she finds herself in because she happens to be the only person that can support Chinese language people and so she will get everything thrown at her, and we are expecting it is a burnout. There is not sufficient funding, full stop. But also, there is not enough commitment to long-term funding ... supporting Chinese migrants is to build capacity ... and you can't build capacity if you are scrambling around for a bit of funding every few months. What you do is you basically just pay for person $X$, but you are not going [to] bother to train them ... because 
you don't know when the next funding's gonna come through. (Peter)

Besides the challenging working situation experienced by the Chinese-languagespeaking staff, overall support for Chinese community organisations was also seen as an issue. It is believed that these organisations can be an important resource for older Chinese migrants simply because there are no language or cultural barriers between the staff and the elderly migrants. Unfortunately, say the practitioners, current support for these Chinese agencies is insufficient:

They [Chinese community agencies] don't have funding. They struggle to get social workers and so we are expecting them to be able to support a community, but we are not supporting them to develop their own capability. And yet it is a vicious circle that continues that you need to do this, but we are not gonna help you in funding. (Peter)

Just as the retired Chinese migrants noted that the language barrier has a significant effect on their daily lives, the aged-services practitioners were also concerned about this issue. Language difficulties are not only limiting retired Chinese migrants' access to social support but also hindering the ability of aged-services practitioners to engage with older Chinese migrants. Furthermore, the perceptions of the service providers around this problem also play an important role in their delivery of social support services.

Due to language difficulties, social support from the Chinese community at large and from the migrant's own family were viewed as crucial resources that can enhance the general well-being of retired Chinese migrants living in Auckland:

I think for the retired Chinese migrants who [are] supported by their family and [who have] good connections within their community ... they are actually well supported, there's a lot of activities and community support to them. (Peter)
The aged-services practitioners all agreed that language difficulties play a key role in the problem of social isolation experienced by retired Chinese migrants in Auckland. If retired Chinese migrants are not connected to a social support service with Chineselanguage-speaking staff, then most would be isolated at home.

Seeing a doctor is another issue when the language barrier creates difficulties:

For example, you get sick, they don't really know which doctor you can see. One had to travel from West to East and she had to wait for one month to see the doctor. (Betty)

One of the practitioners said that difficulties recruiting qualified Chinese-languagespeaking social workers have limited the expansion of support services for retired Chinese migrants:

...because lots and lots of agencies or organisations in New Zealand are quite small, they can't afford to employ Chinese and so some always end up are missing out. (Peter)

While the aged-services practitioners pointed out many of the problems faced by retired Chinese migrants are related to difficulties with the English language, it does not mean that tackling this problem will necessarily and entirely improve the overall well-being of retired Chinese migrants living in Auckland. Their general awareness and understanding of the whole aged-services system in Aotearoa/New Zealand was also a concern. For example:

I had to explain to her [a retired Chinese migrant client] and everything the translation of things. It should be a very simple process but took us over two hours; still couldn't finish it. Although you speak the language for some people, it's more than just translating the information to them. (Peter) 


\section{Comparing findings from both groups}

The findings from both groups, the retired Chinese migrants and the aged-services practitioners, can be compared to highlight any differences for further discussion, because both perspectives are important in understanding the identified needs.

Participants from both groups perceived the language difficulties experienced by retired Chinese migrants in Auckland quite differently. While the migrants saw the problem in terms of their carrying out activities of daily living beyond Chinese speaking environments, the practitioners saw the concerns in relation to professionals' engagement with Chinese migrants. Migrants do not see their difficulty in speaking, reading and listening to English as a problem provided they remain living in Auckland and stay close to the migrant community. This is because most are able to get to Chinese grocery stores and restaurants, choose to see a Chineselanguage-speaking doctor, engage socially in the Chinese community and live in their own ways. The retired Chinese migrants explained that the language barrier becomes most problematic when they are engaging in the health care system in Auckland; for example, seeing a doctor or having treatment in hospital, a concern shared by practitioners in the study.

However, the language problem faced by the older Chinese migrants was perceived by the aged-services providers as a higherranking issue. They said, in their experience, that older migrants who are not able to communicate in English were most likely to be isolated at home and their neighbours might even find it hard to see them. Even if any agencies are willing to provide services to older Chinese migrants, the organisations cannot afford to expand their own current services because of insufficient funding and a shortage of Chinese-speaking practitioners. This clearly suggests that the Chinese community in Auckland is the most important support resource for those
Chinese migrants who face English language barriers. In addition, it may explain why most of the older Chinese migrants do not move out of Auckland. Although speaking Mandarin or Cantonese is fine for people living within the Chinese community in Auckland, moving beyond their boundaried suburb or even community it is commonly agreed to be problematic. This led to a common view amongst practitioners that retired Chinese migrants need help to learn English in order to tackle many different problems in their daily lives, particularly when they need to access health care.

The most interesting finding is the different expectations of aged-support services for Chinese migrants. While the service providers focused more on the sustainability of the service, the service users paid more attention to the way the various services were delivered and who could get support. The retired Chinese migrants said in their focus group discussion that more attention should be paid to the criteria determining who gets support, as well as the quality of the service. As noted above, the migrant participants strongly link their lack of English proficiency to their dependence on family and would also like more funded English language classes where they can learn basic English, so they can cope with issues arising from daily living by themselves, rather than relying on their children.

\section{Discussion}

This study found the English proficiency of older Chinese migrants does not generally meet Immigration New Zealand's expectation of language competency as outlined in its policy. This mismatch is noticeable in the communication difficulties experienced by both the retired Chinese migrants and the practitioners serving them. In fact, all the retired migrants reported difficulties dealing with healthrelated services such as seeing a doctor, staying in hospital, and communicating with health practitioners. Comments 
from the aged-services practitioners give insight into another possible reason for this situation. While the English language level of the retired Chinese migrants in Auckland is in doubt, their overall low education level (including the retired Chinese participants of this study) may also be an obstacle that limits their understanding of what the doctors or nurses are talking about. In other words, even if the retired Chinese migrants can handle basic communication in this country, they may not understand the actual meaning of conversations about diagnosis and treatment with others due to their lack of knowledge in a particular field. This is compounded further when the elderly person's basic English language skills are poor or non-existent. As a result, family or the adult child will be the most significant sources of support for the retired migrant's daily living in Auckland.

In fact, some older migrants were concerned with the eligibility criteria for applying for a place in a retirement village, saying that they do not want to bother their adult child too much. While they did not like the idea of living in a community of aged people and expected they would not find it easy living in a retirement home, the older Chinese migrants felt frustrated with their current situation and wanted an improvement in home-help service. The focus group participants' comments suggest that retired Chinese migrants are experiencing a critical loss of power and independence after moving to a new environment. Very few of them felt confident or able to master the English language, nor did they find it easy to integrate into the local culture. As a result, the retired Chinese migrants felt isolated in their lives with their adult children and reported little social participation in their local communities echoing the findings of Ip et al.'s Brisbane study (2007). Many retired Chinese migrants relied largely on their adult children to cope with tasks that must be performed outside the household and, as a result, feel they lack independence. This, in turn, leads to elderly Chinese migrants feeling burdened by family obligations.
Many of the adult children of these retired Chinese migrants were working full time to support the whole family and so the elderly parents in this study felt they had to, in turn, support their adult child's family through, for example, babysitting and housework. This obligation to their adult child's family constrained their own lives, and the needs of the older parents were sometimes overlooked. In other words, it is argued that the promised family support required by the immigration office is, over time, not an effective way to promote quality of life for retired Chinese migrants.

In addition, the retired Chinese migrant participants indicated during their focus groups that language problems are more problematic when they enter the health system, beyond their Chinese-speaking GPs, for example. Challenges in navigating the health system also takes up much of the aged-services practitioners' time, translating and explaining the situation to the service users who cannot understand or communicate adequately in English. Therefore, due to the current number of retired Chinese migrants in Auckland and the fact that this community is growing, resources are needed to enable the recruitment of on-site translators and Chinese-language-speaking general practitioners in clinics and hospitals. Personnel for both these roles could be recruited from overseas, with a qualification assessment procedure. Referencing Australia's skilled migrants' system, if a Chinese-language-speaking person is qualified to take up the position, under the point-test scale system, Auckland Council could nominate that person to get residency on the grounds they will be using their professional skills to benefit Auckland's Chinese population. Additional requirements could be added, such as the minimum period the applicant needs to stay in Auckland.

In addition to the above, comments from both the elderly Chinese migrants and the social service practitioners in their respective 
focus group interviews suggest the current immigration policy indirectly limits the possibility of retired Chinese migrants living anywhere other than Auckland; for example, in Hamilton or the South Island. The social services practitioners all agreed that the strong Chinese community in Auckland provides significant resources of social support to the Chinese migrants living in that city. With the larger-in-scale community, the retired Chinese migrants can live in their own traditional Chinese way and communicate with Chinese-languagespeaking people. They perceived that, even if retired Chinese migrants were to move to Hamilton, just two hours' drive from Auckland, it would be very difficult for them to adapt to living in Aotearoa New Zealand because there are fewer Chinese stores and restaurants in this Waikato city, the Chinese community is smaller, and there is insufficient support in health services for Chinese-speaking residents. For these reasons, and particularly because of the language barrier, both practitioners and retired Chinese migrants interviewed for this study all agreed that older Chinese migrants faced barriers to moving beyond Auckland city.

The large number of Chinese living in Auckland has already impacted on agedservices practitioners. This study has revealed that some of these practitioners find it stressful assisting retired Chinese migrants, not only because of the time it takes to translate from English to their native tongue, but also because the practitioners have to spend a lot of time explaining the health system to retired Chinese migrants, which the practitioners do not expect to have to do as part of their duties. Moreover, because the government does not fund specifically for the provision of Mandarin- and Cantonesespeaking social workers, when a person is employed who can speak a Chinese language, then they are expected to provide nearly all of the service needs of the retired Chinese migrants no matter whether they are qualified to do the task. This stressful situation is further compounded because the team of the organisation the practitioner works for is generally unable to provide additional support to that staff member.

Auckland's transportation systems were also identified as posing a barrier to retired Chinese migrants' access to social support and other community facilities. Whether the elderly person takes public transport or drives on their own, they will face similar problems when travelling around Auckland, due to communication problems and the other barriers described earlier. It is now imperative that both the government and Auckland Council undertake comprehensive infrastructure planning for the region's fast-growing population, with its diverse communities. An easily navigated mass transport system with a reliable service will enable older people to reach social support from outside their families, particularly retired Chinese migrants.

\section{Conclusions and recommendations}

\section{Support from the Government and community}

More extensive promotion of the government-funded, non-tangible support available for retired Chinese migrants is needed. For example, current support includes free interpreting services, translation services for written words, and free English language classes-but retired Chinese migrants who are facing language barriers need to be made aware of these services.

Therefore, it is reasonable for both Chinese migrants and practitioners to look for more support from the Aotearoa New Zealand government. Much greater and more stable funding would enable service organisations to expand their suppzort for older Chinese migrants by recruiting and training more Mandarin- and Cantonese-speaking social workers, as well as developing more collaborative partnerships between the Chinese agencies and other local organisations. These improvements to the 
delivery of social services will benefit more and more retired Chinese migrants as they are supported in their adaptation to local society.

With the Chinese community being a very important and dominant resource for social support for retired Chinese migrants in Auckland, a good strategy would be to strengthen the Chinese community to become an even more powerful support source. As discussed above, most of the information about the support provided by the government fails to reach the Chinese community and so, even if the Chinese community were approached by a retired Chinese migrant seeking information about, for example, free English language classes, the community would be able to provide only limited help. Thus, more funding to the Chinese community or Chinese service agencies is crucial to enable them to provide social work services. A social worker is assumed to be well trained to provide assistance according to one's specific needs. Under this situation, the social worker can be a social support manager to help the retired Chinese migrants to connect with the different supports they need-for example, to an English language class in the local community.

The government's limited support to agencies that provide social support for retired Chinese migrants is not only a challenge for the Chinese community and small agencies, but also for the relatively larger organisations. Under this challenging situation, collaboration between the organisations would be a way to enhance the services that can be provided for retired Chinese migrants. Given that the Chinese community already has the knowledge of, and a comparatively large network with, retired Chinese migrants, the organisations with funding should engage with the Chinese community and extend their services based on the community's already established strong network. Potentially, there are several key gains from such a collaboration. Firstly, an immediate benefit of collaboration between the funded organisations and the Chinese community would be a reduction in the time currently spent addressing cultural differences between the Chinese service users and the service providers, who are embedded with Western culture. Secondly, in the longer term, collaboration with governmentfunded agencies could empower the Chinese community, helping them to establish services within, and provided by, the local community. When the Chinese community becomes able to manage social support services and is capable of planning service development, they will be more likely to get funding by themselves.

Politicians and policymakers have other challenges in addition to enhancing the Chinese community. The social service providers in this study have identified a need for ongoing training and support for social workers working with nonEnglish-speaking migrants. They reported that any social worker who can speak Mandarin or Cantonese soon becomes overloaded providing support services to the retired Chinese migrants. In the view of the practitioners, some of the needs of the migrants went beyond what the social workers saw their role to be and this, combined with a perceived lack of the support from managers, decreases their job satisfaction and willingness to stay in the position. This is a complex challenge because, even if Chinese-language social workers are recruited to work in Aotearoa New Zealand, they will find it difficult to practise here until they integrate with the local social support system. Placing Chineselanguage-speaking social workers recruited from overseas into positions without supervision and training will not be an effective way to address the current problem. Therefore, it is not merely an issue of labour shortage, but also a result of past failure to comprehensively plan the social support system. Simply speaking, if there is not any planning to enhance and support the service providers, it is hard to move the service forward. Under the current environment, it 
will be difficult to improve social support services for retired Chinese migrants.

\section{Study limitations}

The researcher acknowledges that firstly, this is a small study and it would be unwise to generalise these findings to other ethnic groups in the Auckland region. For pragmatic reasons of time and access in this study, focus groups were a good choice of method of data collection, however, in-depth interviews would be advised to augment groups in further research.

Secondly, this qualitative study is an investigation into retired Chinese migrants' experiences and those of aged-services practitioners in one city in Aotearoa New Zealand. Self-reporting is a reasonable way for study participants to recall their personal experiences, but it does have some disadvantages. Selfreporting means comments cannot be measured against objective standards of perceptions.

Language barriers and community isolation were common concerns of participants in both groups, which implies that future studies should put more effort on exploring the social, cultural and financial needs of older Chinese migrants. More in-depth investigation is needed to explore the needs of this population and to seek their ideas about solutions.

With these caveats in mind, it is recommended that future research should attempt to use a larger sample size of retired Chinese immigrants from different areas of Auckland; this would validate the current study's findings and also make the findings more generalisable. In addition, further research focusing on tackling language barriers experienced by retired Chinese migrants when accessing social support would also be beneficial.

Hence, the researchers strongly believe that it is a fruitful topic for further investigation.
Their connection to a social support network may change over time due to the possible deterioration of their physical ability or other mobility issues. If they become unable to keep in touch with the Chinese community, elderly migrants will be more likely to become isolated from their social support network, and then the elderly migrant will become further isolated or even "disappear" from their community.

\section{Acknowledgements}

The author would like to thank Age Concern Auckland for supporting this study, the participants who shared their experiences and to express thanks for the valuable guidance of my academic supervisors.

Accepted 24 August 2020

Published 15 December 2020

\section{References}

Berry, J. W., Phinney, J. S., Sam, D. L., \& Vedder, P. (2006). Immigrant youth: Acculturation, identity, and adaptation. Applied Psychology, 55(3), 303-332.

Bajekal, M., Blane, D., Grewal, I., Karlsen, S., \& Nazroo, J. (2004). Ethnic differences in influences on quality of life at older ages. Ageing and Society, 24, 709-728.

Chappell, N. L. (2007). Ethnicity and quality of life. In H. Mollenkopf \& A. Walker (Eds.), Quality of life in old age: International and multi-disciplinary perspectives (pp. 179-194). Dordrecht, The Netherlands: Springer.

Cummins, R.A. (2005). Moving from the quality of life concept to a theory. Journal of Intellectual Disability Research, 49(10), 699-706.

Farquhar, M. (1995). Elderly people's definitions of quality of life. Social Science \& Medicine, 41(10), 1439-1446.

Higgs, P., Hyde, M., Arber, S., Blane, D., Breeza, E., Nazzo, J., \& Wiggins, D. (2005). Dimensions of the inequalities in quality of life in older age. In A. Walker (Ed.), Growing older: Understanding quality of life in old age (pp. 27-48). Maidenhead, United Kingdom: Open University Press.

Ip, D., Lui, C. W., \& Chui, W. H. (2007). Veiled entrapment: A study of social isolation of older Chinese migrants in Brisbane, Queensland. Ageing and Society, 27, 719-738.

Li, P. H. (2014). New Chinese immigrants to New Zealand: A PRC dimension. In J. Zhang \& H. Duncan (Eds.), Migration in China and Asia (pp. 229-245). Dordrecht, The Netherlands: Springer.

Remennick, L. (2003). Retired and making a fresh start: Older Russian immigrants discuss their adjustment in Israel. International Migration, 41(5), 153-176. 


\section{ORIGINAL ARTICLE}

QUALITATIVE RESEARCH

Spoonley, P., \& Butcher, A. (2009). Reporting superdiversity. The mass media and immigration in New Zealand. Journal of Intercultural Studies, 30(4), 355-372.

Statistics New Zealand. (2013). 2013 Census. Wellington, New Zealand: Author. Retrieved from http://archive. stats.govt.nz/Census/2013-census/profile-and-summaryreports/quickstats-culture-identity/languag.aspx

Statistics New Zealand. (2015). People aged 65+ living in New Zealand. Wellington, New Zealand: Author. Retrieved from https://www.stats.govt.nz/infographics/ people-aged-65-plus-living-in-new-zealand

Stats NZ. (2017). Ethnic populations projected to grow across New Zealand. Wellington, New Zealand: Author. Retrieved from https://www.stats.govt.nz/news/ethnicpopulations-projected-to-grow-across-new-zealand

Wang, S. C., Schwartz, S. J., \& Zamboanga, B. L. (2010). Acculturative stress among Cuban American college students: Exploring the mediating pathways between acculturation and psychological functioning. Journal of Applied Social Psychology, 40(11), 2862-2887. 